\title{
Diagnosis of gastric cancer by MDCT gastrography: diagnostic characteristics and management potential
}

\author{
Ashraf Anas Zytoon * (D), Safaa Ibrahim Bendary El-Atfey and Shaimaa Abdel-Hamid Hassanein
}

\begin{abstract}
Background: Gastric cancer is regarded as the fifth most frequent tumor globally but the third most common fatal illness. As early as possible, we diagnose cancer stomach especially at early stages, the higher the rate of life. Nevertheless, most cases are diagnosed at late cases where surgery is not of the same benefit at early stages because of clinically indefinite symptoms. The prospective study goal is to estimate the role of MDCT in diagnosis and staging of cancer stomach.

Results: In our study, it was found that there was a high relationship between pathological and CT staging by using MPR. CT with MPR was specific and accurate in diagnosis of all stages of gastric cancer with specificity ranged between 93 and 97\% and accuracy ranged between 90 and 92.5\%. However, it showed lowest sensitivity in diagnosis of stage 1 of gastric cancer. On the other hand, it showed highest sensitivity (90\%) in diagnosis of stage IV as well as we found that MPR and VR of MDCT are much more accurate (92.5\%) than multi-detector computed tomography axial images (80\%) in the diagnosis of all stages of gastric cancer with the difference between the two sequences was significant $(P=0.009)$.

Conclusion: Our results demonstrate that preoperative MDCT with contrast filling technique for abdomen and pelvis evaluates the local disease process of gastric cancer as well as the potential areas of spread. This information is vital in choosing between palliative or radical surgery. MPR and VR help in the assessment of tumor extension and considered as a highly representative prognostic value. Making it the imaging modality of choice in diagnosis and staging of gastric cancers.
\end{abstract}

Keywords: Gastric cancer, Gastric carcinoma, MDCT, MPR

\section{Background}

Gastric cancer is classified as the fifth most common cancer worldwide and the third most common fatal illness [1]. The only available curable treatment is surgical excision, depending on the stage of the disease at the presentation that determined by the extent of stomach wall invasion spread to lymph nodes and multi-organ metastasis. As early as possible we diagnose cancer stomach especially at early stages, the higher the rate of life. Nevertheless, most cases are diagnosed in late cases where surgery is not of the same benefit at early stages because of

\footnotetext{
* Correspondence: ashradio@gmail.com

Radiodiagnosis Department, Faculty of Medicine, Menoufia University, Shebeen El-Kom, Menoufia, Egypt
}

clinically indefinite symptoms. The prospective study goal was to estimate the role of MDCT in the diagnosis and staging of cancer stomach [2].

The extent of wall and peritoneal invasion besides the presence or absence of distant metastasis is more helpful in determining the gastric cancer stages (TNM) [3]. Computed tomography is essentially used for staging of gastric cancer. Advanced techniques such as gastric distension with water or gas have also aided to improve the accurate detection of gastric cancer [4].

Other diagnostic tools as endoscopic ultrasound and MRI can be used in assessing gastric cancer. However, endoscopic ultrasound is an invasive technique, cannot be performed on all patients, is not accurate for the detection of peritoneal disease, and not useful 


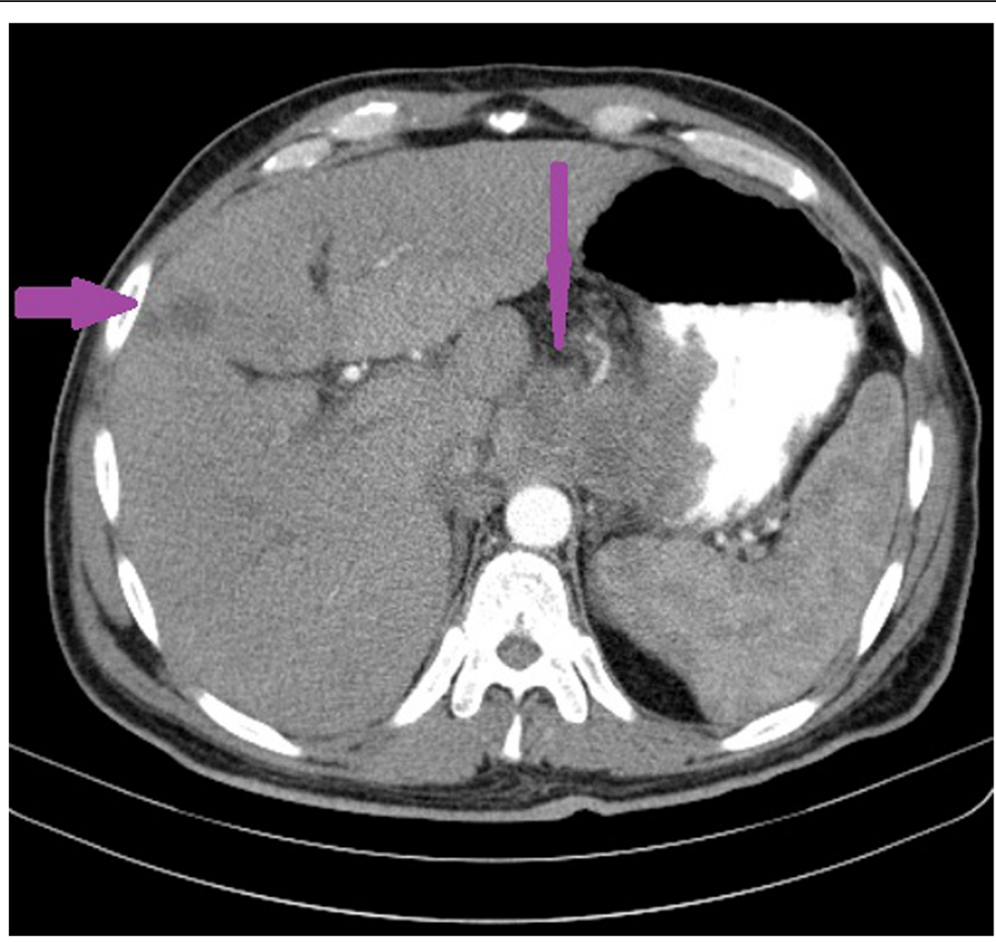

Fig. 1 A 56-year-old male presented with hematemesis for 1 month, a contrast-enhanced CT (arterial phase with oral contrast) axial scans show diffuse relatively enhanced thickness of the lesser curvature reaching gastro esophageal junction, not reaching esophagus, its maximum thickness about $2 \mathrm{~cm}$. Furthermore, there is a hypoechoic round hepatic focal lesion found. Final diagnosis: stage T4NxM1 gastric carcinoma

for detection of distant metastases and although magnetic resonance imaging (MRI) gives higher soft tissue contrast beside the capability of multi-planar imaging, is less used than multi-slice computed tomography because of their prolonged scan time as well as their expensive cost [5].

Preoperative staging is generally performed with abdominal, endoscopic ultrasonography in combination with computed tomography [6]. Currently, endoscopic radiological imaging was recorded as the most useful diagnostic radiological method of preoperative staging to assess the extent of the tumor spread $[6,7]$. The most recent global consent verified the importance of preoperative TNM staging and specified multi-slice computed tomography as the best staging radiological technique $[8,9]$, which has displayed identical or higher accuracy in comparison with endoscopic ultrasonography for $\mathrm{T}$-staging and a clear benefit concerning alternative techniques for TNM staging. Our prospective study goal is to estimate the role of MDCT in the diagnosis and staging of cancer stomach.

\section{Patient and methods}

The present study was conducted between October 2017 and October 2018 on 40 patients (25 males and 15
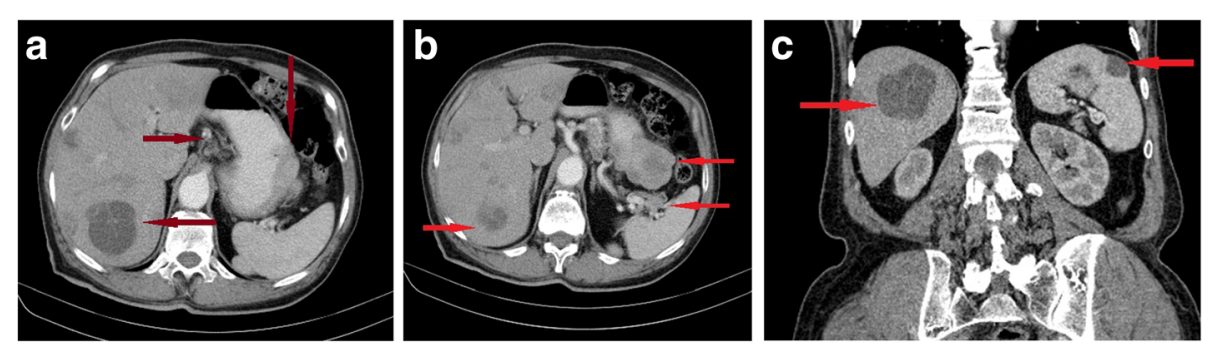

Fig. 2 A 50-year-old male presented with vomiting of blood and dyspepsia for 3 weeks; a, b contrast-enhanced CT (arterial phase with oral contrast) axial scans show diffuse thickness of the greater curvature with an exophytic mass showing heterogeneous enhancement with central breakdown, the mass measures about $(3.5 \times 2.0 \mathrm{~cm})$, other findings are perigastric, splenic hilar lymph nodes and multiple hepatic focal lesion. c Contrast-enhanced CT abdomen coronal scans show splenic and hepatic focal lesions. Final diagnosis: stage T4N1M2 gastric carcinoma 
Table 1 Shape of gastric cancer by MDCT

\begin{tabular}{|c|c|c|c|c|c|c|}
\hline \multirow{2}{*}{$\begin{array}{l}\text { Shape of gastric cancer by } \\
\text { MDCT }\end{array}$} & \multicolumn{2}{|c|}{ Male } & \multicolumn{2}{|c|}{ Female } & \multicolumn{2}{|c|}{ Total } \\
\hline & N & $\%$ & $N$ & $\%$ & N & $\%$ \\
\hline Polypoidal mass & 1 & 2.5 & 1 & 2.5 & 2 & 5 \\
\hline Fungating mass & 2 & 5 & 1 & 2.5 & 3 & 7.5 \\
\hline Ulcerating lesion & 1 & 2.5 & 1 & 2.5 & 2 & 5 \\
\hline Circumferential wall thickening & 21 & 52.5 & 12 & 30 & 33 & 82.5 \\
\hline Total & 25 & 62.5 & 15 & 37.5 & 40 & 100 \\
\hline
\end{tabular}

females) with age ranged from 35 to 75 years with mean age of 55 years. The patients were complaining of symptoms of gastric cancer, referred to the radiological department at Tanta Cancer Institute. This study was approved by our Institutional Review Board.

\section{Inclusion criteria}

In our study, we included patients suffered from recurrent attacks of hematemesis, dyspepsia, of high clinical suspicious of gastric tumors as well as patients presented with untreatable anemia.

\section{Exclusion criteria}

In our study, we excluded pregnant or lactating women as well as patients with renal impairment (elevated serum creatinine value above the standard level), cardiac failure, and patients with history of previous allergic reaction to contrast agents.

\section{Patient preparation and positioning}

Patients were instructed to fast for more than $8 \mathrm{~h}$. Each patient received an intramuscular administration of $20 \mathrm{mg}$ of anisodamine to decrease peristaltic bowel movement and drink $1000-1200 \mathrm{ml}$ plain water for gastric filling $10-20 \mathrm{~min}$ before the start of the scan. Patients were prepared for examination in a supine position.

\section{Contrast material injection}

After an un-enhanced scan of the upper abdomen, a $100 \mathrm{ml}$ bolus of nonionic iodine contrast agent was administered into the antecubital vein with an automatic injector is needed for contrast injection at a flow rate of $3 \mathrm{ml} / \mathrm{s}$ via a 20 -gauge needle using. CT acquisitions were

Table 2 Anatomical location of the gastric mass detected by MDCT

\begin{tabular}{lll}
\hline Anatomical location of the gastric mass detected by MDCT & No. & $\%$ \\
\hline Fundus & 2 & 5 \\
Greater curvature & 17 & 42.5 \\
Lesser curvature & 18 & 45 \\
Pylorus & 3 & 7.5 \\
Total & 40 & 100 \\
\hline
\end{tabular}

Table 3 The sites for local spread and distant metastasis associated with gastric cancer

\begin{tabular}{lcc}
\hline $\begin{array}{l}\text { The sites for local spread and distant metastasis associated } \\
\text { with gastric cancer }\end{array}$ & No. $\%$ \\
\hline Liver & 20 & 55.6 \\
Gastric and perigastric lymph nodes & 12 & 33.3 \\
Esophagus & 4 & 11.1 \\
Total & 36 & 100 \\
\hline
\end{tabular}

performed in the arterial phase (start delay of $30 \mathrm{~s}$ ), in the portal venous phase (start delay of $75 \mathrm{~s}$ ), and the equilibrium phase (start delay of $180 \mathrm{~s}$ ). At the time of MDCT scanning, we restricted arterial and portal venous phase acquisition to the stomach with a section thickness of $3.75 \mathrm{~mm}$.

\section{Scanning criteria}

The examination comprises the abdomen and pelvis with a slice thickness of $5 \mathrm{~mm}$, from the dome of the diaphragm to the anal level. CT scanning guidelines were as follows: 128 detector rows used; pitch, 3; reconstruction interval, $2.5 \mathrm{~mm} ; 200 \mathrm{~mA} ; 120 \mathrm{kV}$; and tube rotation time, $0.8 \mathrm{~s}$.

\section{Post-scanning processing}

All CT images were transmitted to a workstation for multiplanar reconstruction (MPR). The depth of tumor invasion was detected by a plane vertical to the tumor to avoid partial volume effects. These MPR images had a section thickness of $2.5 \mathrm{~mm}$ and were obtained at $2.5-\mathrm{mm}$ intervals.

\section{Results}

In our study, all 40 patients were primarily evaluated by the MSCT protocol before beginning of any treatment therapy as it was important to be firstly diagnosed and staged for estimation of the probable treatment protocol whether surgical or therapeutic. The most frequently involved age group was the group $>50-70$ years and more common in males. The most frequently encountered clinical presentations among gastric carcinoma patients

Table 4 Comparing the MDCT (T) stage with the pathological (T) stage

\begin{tabular}{lllll}
\hline $\mathrm{CT}$ & \multicolumn{4}{l}{ Pathological } \\
\cline { 2 - 5 } Staging & PT1 $(n=2)$ & PT2 $(n=4)$ & PT3 $(n=13)$ & PT4 $(n=21)$ \\
\hline T1 $(n=4)$ & 1 & 2 & 1 & - \\
T2 $(n=4)$ & 1 & 2 & 1 & - \\
T3 $(n=10)$ & - & - & 9 & 1 \\
T4 $(n=22)$ & - & - & 2 & 20 \\
$X^{2}$ & 4.526 & & & \\
$P$ value & 0.011 & & & \\
\hline
\end{tabular}


were vomiting after meal and loss of appetite (Figs. 1 and 2).

Our study observed the correlation between the CT imaging data and pathological data in all 40 patients; it was found that the undifferentiated adenocarcinoma is the most common gastric cancer (Table 1).

All 40 patients were scanned by MDCT, greater and lesser curvature were involved in 35 patients (Table 2). In our study, the commonest site for metastasis was hepatic metastasis (Table 3). Most of our patients had stage T4 $(N=20)$ according to CT staging of gastric carcinoma using MPR and pathological staging. The difference between the CT staging and pathological staging of gastric cancers was significant (Table 4), (Figs. 3 and 4).

Regarding tumor staging by using axial cuts, MPR, and VR sequences, stage 4 of the cancer stomach was better recognized by using MPR and VR sequences, while grade II and grade III of cancer stomach was better identified by using axial cuts with thin slice MDCT. However, the difference between the two sequences was of quarry significance ( $P$ less than 0.05 ) (Table 5 , Fig. 5 ).

\section{Discussion}

Cancer stomach is considered the third most common lethal disease worldwide [1]. Although the usage of double contrast study helps in early detection of any small gastric lesions, wall invasion extent as well as presence or absence of distant metastasis [10]. The definite function of MDCT is to differentiate between benign and malignant gastric tumor and assess the stage and metastatic extent of gastric carcinoma, for assessing treatment protocol between palliative or radical surgical treatment. In our study, the use of contrast agents is essential as there must be adequate distension of the stomach. If the entire stomach is not well distended, disease must be over looked or, conversely, the collapsed gastric wall may mimic disease [10].

On the other hand, MDCT help in evaluation of treatment response. It is also considered as one of the important prognostic factors through tumor extent evaluation [11]. In our study, 40 patients underwent analytical study, correlation, and comparison of MDCT radiological data with histopathologically proved results.

We found male patients were more affected (25/ 40) $(62.5 \%)$ in comparison with female patients (15/ 40) (37.5\%), that undergoes with Macdonald et al. [11], who found that gastric cancer are more common in male patients in comparison to female patients.

The majority of patients had complaints at the diagnosis time of the previous study of Allum et al. [12], who found that dyspepsia, dysphagia, weight loss, and anemia were the most common clinical presentations. This coincides with the findings in which the most frequent clinical presentation was vomiting after a meal in 30 patients followed by loss of appetite in 20 patients, weight loss in 9 patients, and epigastric pain in 6 patients.

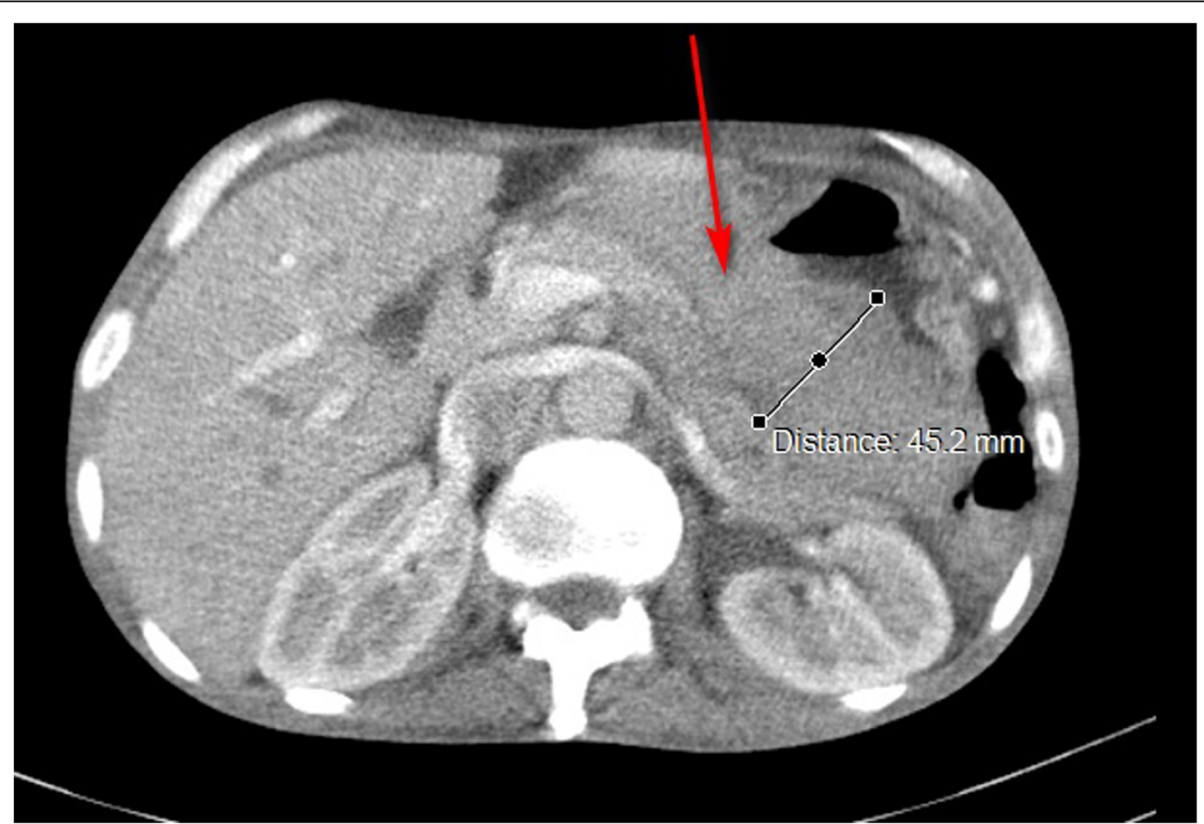

Fig. 3 A 49-year-old female presented with hematemesis for 1 month; a contrast-enhanced CT (arterial phase with oral contrast) axial scans show diffusely enhanced greater curvature mass lesion measures about $4.5 \mathrm{~cm}$ with no regional lymph node spread nor metastasis either locally or distantly Final diagnosis: stage T3NOMO gastric carcinoma 

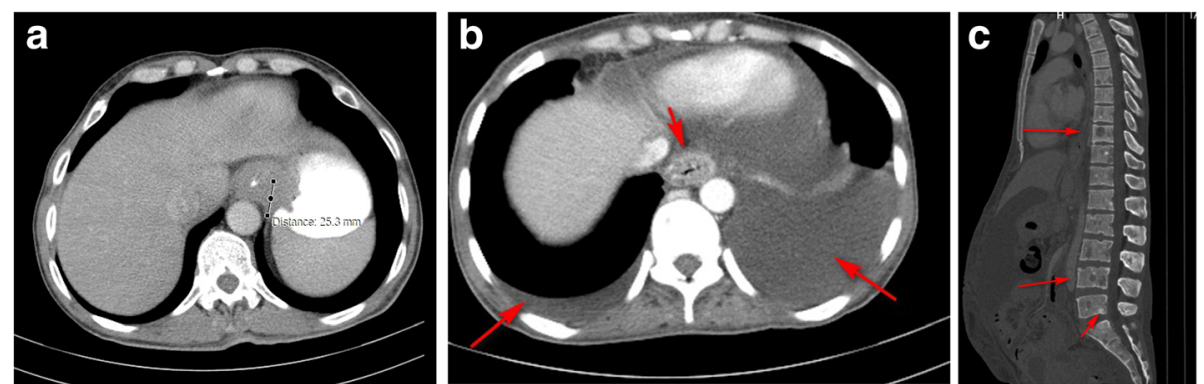

Fig. 4 a A 66-year-old male presented with chronic anemia; a contrast-enhanced CT (arterial phase with oral contrast) axial scans showing diffuse thickness of the gastro esophageal junction reaching the lower esophagus with maximum thickness about $2.5 \mathrm{~cm}$. b Contrast-enhanced CT arterial phase mediastinal window axial scans show bilateral pleural effusion more at the left side with no pulmonary nodules detected. $\mathbf{c} C T$ abdomen sagittal scans bone window showing multiple lytic and sclerotic bony metastasis of the spine. Final diagnosis: stage T4NxM1 gastric carcinoma

According to Perez and Brady [13] who found that $50 \%$ of study cases were with focal mural thickness, $25 \%$ were with polypoidal soft tissue lesions but only $5 \%$ show diffuse mural thickening with narrowing of the lumen and that is in contrary with our results that found about 33 patients showed circumferential gastric wall thickening (82.5\%) but in few cases 3 and 2 patients (7.5\% \& $5 \%)$ polypoid, fungating, and ulcerating masses were found.

According to Horton and Fishman [14] analysis who found that the liver was the most common organ for distant metastasis and in our result there is a high degree of agreement as about half of the study cases metastasized to liver followed by regional L. Ns metastasis and peritoneal deposits.

The stomach layers are best evaluated in the arterial phase of contrast enhancement when the stomach is free of contrast [15]. In our study, gastric tumor enhancement was identified in all patients in the arterial phase, all of them show homogenous enhancement. On MDCT, the extension of tumor load was categorized as follows: T0, no proof of alteration of the gastric wall with even perigastric fat around; T1, infiltration of the gastric mucosa or submucosa [16]; T2, invasion to muscularis propria [17];

Table 5 The tumor detection rate with MDCT axial cuts, MPR, and VR in different stages

\begin{tabular}{|c|c|c|c|c|}
\hline \multirow{3}{*}{$\begin{array}{l}\text { Cancer } \\
\text { staging } \\
\text { by } \\
\text { MDCT }\end{array}$} & \multicolumn{4}{|c|}{ Number of patients detected in different stages either with } \\
\hline & \multicolumn{2}{|c|}{ MPR and volume rendering } & \multicolumn{2}{|c|}{ Axial cuts } \\
\hline & N & $\%$ & N & $\%$ \\
\hline $\mathrm{T1}$ & 3 & 7 & 2 & 5 \\
\hline T2 & 5 & 12.5 & 4 & 10 \\
\hline T3 & 11 & 27.5 & 10 & 25 \\
\hline T4 & 21 & 52.5 & 20 & 50 \\
\hline$x^{2}$ & 0.637 & & & \\
\hline$P$ value & 0.115 & & & \\
\hline
\end{tabular}

T3, invasion to subserosa [16]; and T4, invasion to serosa and adjacent organs or structures $[18,19]$.

In the present study, the sensitivity of MDCT in recognition and evaluation of gastric neoplasms was documented parallel with the histopathological results as a gold standard. In the current study, there is a significant relationship between pathological and CT staging by using of thin-slice axial CT as we found that CT was specific and accurate in diagnosis of all stages of gastric cancer with specificity ranged between 93 and $97 \%$ and accuracy ranged between 9 and $92.5 \%$.

The present study showed that MDCT gives the highest sensitivity (90\%) in stage IV, but the lowest on of stages I and II. This correlates with Kumano et al. [20] results, who reported that MDCT has sensitivities in the range of 68.8$96.2 \%$ in the detection of gastric cancers. According to our recent statistics, the accuracy and sensitivity of T3 are $70 \%$ and $100 \%$, while that of $\mathrm{T} 4$ was 70 and 44 , respectively.

There are points of strengths in the current study; as our study was done on a tertiary cancer center promoting the possibility of checking as many suspecting cases with follow up, using the advanced MDCT machine of 128-row detectors giving the chance for more accuracy and detection of small lesions with its first metastasis. Furthermore, the detection of our patients with gastric carcinoma included different stages and detection of different metastasis unpredicted sites either presented as local tumor spread or in distant sites. Finally, our study correlates all the MDCT findings with the histopathological results giving more confidence.

On the contrary, there are few points of limitations could not be evaded and should be recorded; our patient sample is limited, but inevitably our next research will include large sample in multi-centric study, most cases were presented in advanced stage, and deficiency of post-operative or post-therapy follow up to show the impact of our results upon the management plan but another research could be intensive study that point. 

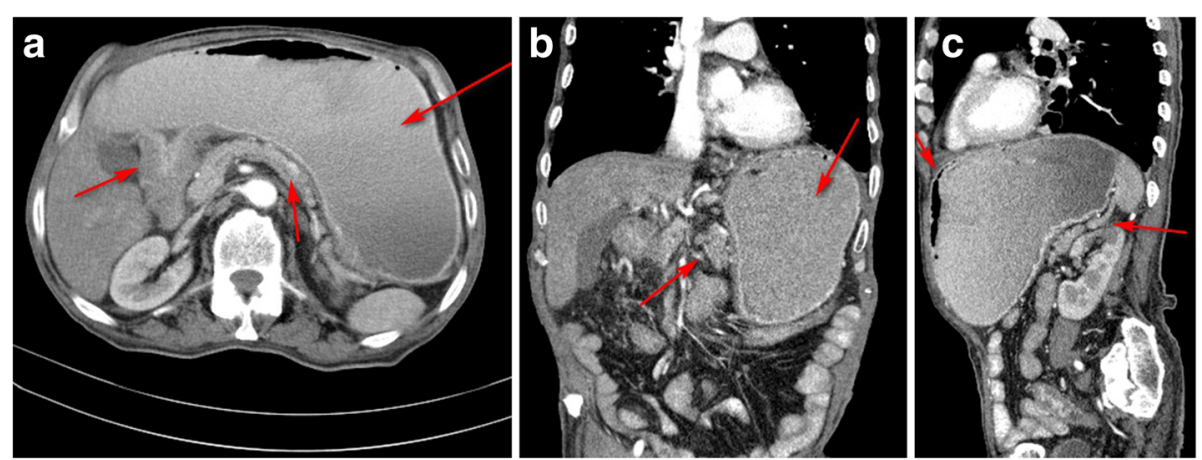

Fig. 5 (a, b) A 67-year-old male presented with hematemesis for 2 weeks, contrast enhanced CT (arterial phase with oral contrast) axial scans show markedly distended stomach with diffuse pyloric thickening causing slit like lumen, perigastric chain of lymph nodes. (c) also confirming the presence of markedly distended stomach with chain of perigastric lymph nodes. Final Diagnosis : Stage T3N1M0 with subsequent gastric outlet obstruction

\section{Conclusion}

Our results demonstrate that preoperative MDCT with contrast filling technique for abdomen and pelvis evaluates the local disease process of gastric cancer as well as the potential areas of spread. This information is vital in choosing between palliative or radical surgery. MPR and VR help in the assessment of tumor extension and considered as a highly representative prognostic value. Making it the imaging modality of choice in diagnosis and staging of gastric cancers, as the MDCT (using the contrast filling technique) with added MPR and VR images is definite in $92.5 \%$ but of $95 \%$ sensitivity in proper diagnosis, staging as well as follow-up of patients with cancer stomach.

\section{Abbreviations}

3D: Three dimensional; CT: Computed tomography; MDCT: Multi-detector computed tomography; MPR: Multi-planar reconstruction; MRI: Magnetic resonance imaging; M-stage: Metastases stage; $\mathrm{N}$-stage: Lymph node stage; TNM staging: Primary tumor, regional lymph nodes, distant metastasis; Tstage: Tumor stage; VR: Volume rendering

\section{Acknowledgements}

The authors acknowledge those who have contributed substantially to the work, such as editorial and writing assistance, but who are not included as authors should also be included.

Our marvelous appreciation and thanks to the soul of Prof. Dr. Adel Mohammed Alwakeel for his encouragement, sustained support, expert guidance, valuable instructions, and suggestions.

\section{Authors' contributions}

$A A Z$, SIBE-A, and SA-HH contributed to the conception and design of the study, acquisition, analysis and interpretation of data, creation of the final work, revision, and accuracy or integrity of the submitted manuscript. All authors have read and approved the manuscript.

\section{Funding}

None.

\section{Availability of data and materials}

The datasets used and/or analyzed during the current study are available from the corresponding author on reasonable request.
Ethics approval and consent to participate

Our study was approved by the ethical and scientific committee of Faculty of Medicine, Menoufia University. Written consent to participate ref. no. 2017/8/12/1278

\section{Consent for publication}

Not applicable.

\section{Competing interests}

The authors declare that they have no competing interests.

Received: 30 October 2019 Accepted: 23 January 2020

Published online: 07 February 2020

\section{References}

1. Virgilio E, D'Antonio C, Balducci G (2014) Meso gastrium recurrence as expression of the fifth metastatic route of gastric cancer. Med Hypotheses 82(3):403

2. Sharara SM, Nagi MA, Soliman SS (2018) Multidetector computed tomography in the evaluation of gastric malignancy; a multicenteric study. The Egyptian Journal of Radiology and Nuclear Medicine 49(2):304-309

3. Yogaraj S, Kumar MS (2019) 128 slices multidetector CT evaluation of gastric carcinoma - imaging and histopathological correlation. IAIM 6(4):60-63

4. Thrumurthy SG, Chaudry MA, Hochhauser D (2013) The diagnosis and management of gastric cancer. Br Med J 347:6367

5. Liu S, Liu S, Ji C, Zheng H, Pan X, Zhang Y, Guan W, Chen L, Guan Y, Li W, He J (2017) Application of CT texture analysis in predicting histopathological characteristics of gastric cancers. Eur Radiol 27(12):49514959

6. Bray F, Ferlay J, Soerjomataram I, Siegel RL, Torre LA, Jemal A (2018) Global cancer statistics 2018: GLOBOCAN estimates of incidence and mortality worldwide for 36 cancers in 185 countries. CA Cancer J Clin 68(6):394-424

7. Almeida MFA, Verza L, Bitencourt AGV, Boaventura CS, Barbosa PNVP, Chojniak R (2018) Computed tomography with a stomach protocol and virtual gastroscopy in the staging of gastric cancer: an initial experience. Radiol Bras 51(4):211-217

8. Coburn N, Seevaratnam R, Paszat L (2014) Optimal management of gastric cancer: results from an international RAND/UCLA expert panel. Ann Surg 259:102-108

9. Dixon M, Cardoso R, Tinmouth J, Helyer L, Law C, Swallow C, Paszat L, McLeod R, Seevaratnam R, Mahar A, Coburn NG (2014) What studies are appropriate and necessary for staging gastric adenocarcinoma? Results of an international RAND/UCLA expert panel. Gastric Cancer 17(2):377-382

10. Teama AH, El-Badry AM, Yousef ES (2016) The role of multislice computed tomography in the diagnosis of gastric malignant tumors. Tanta Med J 44 119-126

11. Macdonald JS, Hundahl S, Smalley SR, O'Dea D, Mitchell EP (2011) Gastric cancer. In: Gastrointestinal Oncology, pp 101-138 
12. Allum WH, Blazeby JM, Griffin SM, Cunningham D, Jankowski JA, Wong R (2011) Guidelines for the management of oesophageal and gastric cancer Gut 60(11):1449-1472

13. Perez CA, Brady LW (2008) Principles and practice of radiation oncology. Lippincott Williams \& Wilkins, Philadelphia, PA, pp 620-636

14. Horton KM, Fishman EK (2003) Current role of CT in imaging of the stomach. Radio graphics 23(1):75-87

15. Brasil. Ministério da Saúde (2016) Instituto Nacional de Câncer José Alencar Gomes da Silva. Estimativa/2016: incidência de câncer no Brasil. INCA, Rio de Janeiro, RJ

16. Barros RH, Penachim TJ, Martins DL, Andreollo NA, Caserta NM (2015) Multidetector computed tomography in the preoperative staging of gastric adenocarcinoma. Radiol Bras 48(2):74-80

17. Kim JW, Shin SS, Heo SH, Lim HS, Lim NY, Park YK, Jeong YY, Kang HK (2015) The role of three-dimensional multidetector CT gastrography in the preoperative imaging of stomach cancer: emphasis on detection and localization of the tumor. Korean J Radiol 16(1):80-89

18. Lee DH, Kim SH, Joo I, Han JK (2018) CT perfusion evaluation of gastric cancer: correlation with histologic type. Eur Radiol 28(2):487-495

19. Emam HM, Moussa EM, Abouelmaged M, Ibrahim MR (2019) Role of multidetector $C T$ in staging of gastric carcinoma. Journal of Cancer Therapy 10:565-579

20. Kumano S, Okada M, Shimono T et al (2012) T-staging of gastric cancer of air-filling multidetector-row CT: comparison with hydro-multidetector row CT. Eur J Radiol 81:2953-2960

\section{Publisher's Note}

Springer Nature remains neutral with regard to jurisdictional claims in published maps and institutional affiliations.

\section{Submit your manuscript to a SpringerOpen ${ }^{\circ}$ journal and benefit from:}

- Convenient online submission

- Rigorous peer review

- Open access: articles freely available online

- High visibility within the field

Retaining the copyright to your article

Submit your next manuscript at $\boldsymbol{\nabla}$ springeropen.com 